\title{
Geometric Aspects of Space-Time Reflection Symmetry in Quantum Mechanics
}

\author{
Carl M. Bender, Dorje C. Brody, Lane P. Hughston, and Bernhard K. Meister
}

\begin{abstract}
For nearly two decades, much research has been carried out on properties of physical systems described by Hamiltonians that are not Hermitian in the conventional sense, but are symmetric under space-time reflection; that is, they exhibit $\mathscr{P} \mathscr{T}$ symmetry. Such Hamiltonians can be used to model the behavior of closed quantum systems, but they can also be replicated in open systems for which gain and loss are carefully balanced, and this has been implemented in laboratory experiments for a wide range of systems. Motivated by these ongoing research activities, we investigate here a particular theoretical aspect of the subject by unraveling the geometric structures of Hilbert spaces endowed with the parity and time-reversal operations, and analyze the characteristics of $\mathscr{P} \mathscr{T}$-symmetric Hamiltonians. A canonical relation between a $\mathscr{P} \mathscr{T}$-symmetric operator and a Hermitian operator is established in a geometric setting. The quadratic form corresponding to the parity operator, in particular, gives rise to a natural partition of the Hilbert space into two halves corresponding to states having positive and negative $\mathscr{P} \mathscr{T}$ norm. Positive definiteness of the norm can be restored by introducing a conjugation operator $\mathscr{C}$; this leads to a positive-definite inner product in terms of $\mathscr{C} \mathscr{P} \mathscr{T}$ conjugation.
\end{abstract}

Carl M. Bender

Department of Physics, Washington University, St. Louis MO 63130, USA,

e-mail: cmb@wustl.edu

Dorje C. Brody and Lane P. Hughston

Department of Mathematics, Brunel University London, Uxbridge UB8 3PH, UK

and Department of Optical Physics and Modern Natural Science, ITMO, 49 Kronverksky Avenue,

St Petersburg 197101, Russia

e-mail: dorje.brody@brunel.ac.uk and e-mail: lane.hughston@brunel.ac.uk

Bernhard K. Meister

Department of Physics, Renmin University of China, Beijing, China 10087

e-mail: bernhard@fasteagle.jp 


\section{Introduction}

The observation that non-Hermitian Hamiltonians possessing a physical symmetry associated with a discrete space-time reflection, known as $\mathscr{P} \mathscr{T}$ invariance, can possess entirely real eigenvalues [1] has generated considerable research outputs for nearly two decades. It is by now well documented that a $\mathscr{P} \mathscr{T}$-symmetric Hamiltonian possesses real eigenvalues if the symmetry is unbroken in the sense that eigenstates of $H$ are also eigenstates of $\mathscr{P} \mathscr{T}$ [2]. A $\mathscr{P} \mathscr{T}$-symmetric Hamiltonian can describe dynamical and probabilistic aspects of closed quantum systems if one augments the Hilbert space with a suitable inner product [3, 4]. A $\mathscr{P} \mathscr{T}$ symmetric Hamiltonian can be replicated in an open system by balancing gain and loss $[5,6,7]$. Open systems can have phase transitions associated with the breaking of $\mathscr{P} \mathscr{T}$ symmetry, leading to a range of counterintuitive phenomena that have been observed in laboratory experiments for many different kinds of physical systems $[8,9,10,11,12,13,14,15]$.

For a quantum system having continuous degrees of freedom parity reflection $\mathscr{P}$ has the classical analog $x \rightarrow-x$ and $p \rightarrow-p$. Time reversal $\mathscr{T}$ generates the transformations $p \rightarrow-p$ and $\mathrm{i} \rightarrow-\mathrm{i}$. In the case of an open system modeled on a finite-dimensional Hilbert space $\mathscr{P}$ can be interpreted as the interchange of the left and the right sides of the system and $\mathscr{T}$ amounts to interchanging the gain and loss channels. Hence, if the mirror image of the gain channel is a loss channel, then $\mathscr{P} \mathscr{T}$ symmetry can be realized if the strengths of gain and loss are matched exactly. For a closed system characterized by a finite-dimensional matrix Hamiltonian the interpretation of $\mathscr{P}$ is not immediately apparent. Nevertheless, one can augment the Hilbert space with a structure that in a general sense embodies properties of parity reflection. It is then of interest to investigate the mathematical properties of Hilbert spaces endowed with such a structure.

This paper addresses this question by clarifying mathematical, and in particular the geometric aspects of the underlying real Hilbert space endowed with the parity structure. Apart from its intrinsic mathematical appeal, the geometric formalism has led, even in standard Hermitian quantum theory, to discoveries that no other mathematical approach has reproduced (for example, higher-order corrections to the Heisenberg uncertainty relation $[16,17])$, or discoveries that come naturally with the geometric formalism (for example, the identification of the measure of entanglement for pure states [18]). In this spirit we develop here a geometric framework that is sufficiently general to admit both standard quantum theory with a Hermitian Hamiltonian as well as extensions of the standard theory. In Secs. 2 and 3 we discuss the underlying mathematical structures and the role of the observables in conventional Hermitian quantum mechanics. In Secs. 4 and 5 we compare these results in with the corresponding structures in $\mathscr{P} \mathscr{T}$-symmetric quantum theory. It is well known that the requirement of $\mathscr{P} \mathscr{T}$ invariance alone on the Hamiltonian leads to a state space with an indefinite metric. The crucial observation that we make here is that the parity operator plays the role of an indefinite metric, while the complex structure $J$ of standard quantum mechanics is unaltered in $\mathscr{P} \mathscr{T}$-symmetric quantum theory. This is an attractive complex-analytic feature of $\mathscr{P} \mathscr{T}$-symmetric theory. 
Proposition 1 states that the $\mathscr{P} \mathscr{T}$ norm of a state is expressible as a difference of the standard Dirac norm of the positive- and negative-parity parts of the state.

Section 6 analyses the properties of $\mathscr{P} \mathscr{T}$-symmetric Hamiltonian operators. Proposition 2 shows that any such Hamiltonian is a product of the parity structure and a Hermitian quadratic form. This leads to a new way to understand the reality of the spectrum of such Hamiltonians; Proposition 3 shows that the energy eigenvalues are necessarily real if the corresponding eigenvectors have nonvanishing $\mathscr{P} \mathscr{T}$ norm. In our geometric scheme Proposition 4 shows that the eigenvalues of such Hamiltonians are either real or occur as complex conjugate pairs [19]. A sufficient condition for the reality of the eigenvalues is then established in Proposition 5.

In Sec. 7 we introduce an additional structure $\mathscr{C}$ that has the interpretation of charge conjugation. This symmetry allows us to construct an alternative inner product on the vector space spanned by the eigenfunctions of the $\mathscr{P} \mathscr{T}$-symmetric Hamiltonian by means of $\mathscr{C} \mathscr{P} \mathscr{T}$ conjugation, thus eliminating states having negative norms. As a consequence, a consistent probabilistic interpretation for a closed system can be assigned to quantum theories described by $\mathscr{P} \mathscr{T}$-symmetric Hamiltonians.

\section{Geometry of Hermitian quantum mechanics}

Before discussing $\mathscr{P} \mathscr{T}$-symmetric quantum theory, it is helpful first to formulate standard quantum mechanics from a perspective that is useful in clarifying the similarities and differences of the two formalisms. In standard quantum theory Hermitian operators play a dual role, namely, as physical observables and as generators of dynamics. To explain the relation between these two roles, we show how to build quantum mechanics, not in terms of the complex Hilbert space with respect to which it is usually formulated, but rather in terms of a more primitive underlying even-dimensional real Hilbert space $\mathbb{H}$. By introducing certain structures on $\mathbb{H}$ we arrive at standard quantum theory. Then by considering a related alternative set of structures on $\mathbb{H}$ we arrive at $\mathscr{P} \mathscr{T}$-symmetric quantum theory, and the relationship of the two theories becomes clear.

Using index notation [Geroch [20], Gibbons and Pohle [21], Wald [22], Brody and Hughston [16, 17]], we let the real vector $\xi^{a}$ denote a typical element of $\mathbb{H}$. The real Hilbert space $\mathbb{H}$ is equipped with a positive-definite quadratic form $g_{a b}$ satisfying $g_{a b}=g_{b a}$, and the squared norm of the vector $\xi^{a}$ is given by $g_{a b} \xi^{a} \xi^{b}$. If $\xi^{a}$ and $\eta^{a}$ are elements of $\mathbb{H}$ we define their inner product by $g_{a b} \xi^{a} \eta^{b}$.

To recover the apparatus of standard quantum mechanics we require that $\mathrm{H}$ also be endowed with a compatible complex structure, by which we mean a real tensor $J_{b}^{a}$ whose square is equal to the negative of the identity $J_{c}^{a} J_{b}^{c}=-\delta_{b}^{a}$. The complex structure is compatible with the symmetric quadratic form if $g_{a b}$ satisfies

$$
g_{a b} J_{c}^{a} J_{d}^{b}=g_{c d}
$$


and $g_{a b}$ is said to be $J$-invariant. The $J$-invariance condition implies that the tensor

$$
\Omega_{a b}=g_{a c} J_{b}^{c}
$$

is antisymmetric and nondegenerate, and thus defines a symplectic structure on H. To verify the antisymmetry of $\Omega_{a b}$ we insert (1) into (2): $\Omega_{b a}=g_{b c} J_{a}^{c}=$ $g_{d e} J_{b}^{d} J_{c}^{e} J_{a}^{c}=-g_{d e} J_{b}^{d} \delta_{a}^{e}=-\Omega_{a b}$.

To verify the nondegeneracy of $\Omega_{a b}$ we note that $\Omega^{a b}=g^{a c} g^{c d} \Omega_{c d}$ acts as the required inverse. Indeed, we have $\Omega^{a c} \Omega_{b c}=g^{a e} g^{c f} g_{e h} J_{f}^{h} g_{b d} J_{c}^{d}=g_{b d} J_{c}^{d} J_{f}^{a} g^{c f}=\delta_{b}^{a}$. In the last step we have used the $J$-invariance of $g^{a b}$, which satisfies $J_{c}^{a} J_{d}^{b} g^{c d}=g^{a b}$. The symplectic structure is also compatible with $J_{b}^{a}$ in the sense that $\Omega_{a b} J_{c}^{a} J^{b}{ }_{d}=$ $\Omega_{c d}$. (This follows because $\Omega_{a b} J_{c}^{a} J^{b}{ }_{d}=g_{a e} J_{b}^{e} J_{c}^{a} J^{b}{ }_{d}=-g_{a e} \delta_{d}^{e} J_{c}^{a}=-\Omega_{d c}=\Omega_{c d}$.) We then say that $\Omega_{a b}$ is $J$-invariant.

We can now elucidate the structure of standard quantum mechanics. We endow the real Hilbert space $\mathbb{H}$ with a Hermitian inner product. If $\xi^{a}$ and $\eta^{a}$ are two real Hilbert space vectors, then their Hermitian inner product, which we write as $\langle\eta \mid \xi\rangle$ in the usual Dirac notation, is the complex expression

$$
\langle\eta \mid \xi\rangle=\frac{1}{2} \eta^{a}\left(g_{a b}-\mathrm{i} \Omega_{a b}\right) \xi^{b} .
$$

Because the symplectic form $\Omega_{a b}$ is antisymmetric, the Hermitian norm agrees with the real Hilbertian norm, apart from a factor of two: $\langle\xi \mid \xi\rangle=\frac{1}{2} g_{a b} \xi^{a} \xi^{b}$.

Next, we complexify the Hilbert space $\mathbb{H}$ and denote the result $\mathbb{H}_{\mathbb{C}}$. The elements of $\mathbb{H}_{\mathbb{C}}$ are the complex vectors $\xi^{a}+\mathrm{i} \eta^{b}$, where $\xi^{a}, \eta^{b} \in \mathbb{H}$. With the aid of the complex structure, a real Hilbert space vector $\xi^{a}$ can be decomposed into complex $J$-positive and $J$-negative parts:

$$
\xi^{a}=\xi_{+}^{a}+\xi_{-}^{a},
$$

where

$$
\xi_{+}^{a}=\frac{1}{2}\left(\xi^{a}-\mathrm{i} J_{b}^{a} \xi^{b}\right) \quad \text { and } \quad \xi_{-}^{a}=\frac{1}{2}\left(\xi^{a}+\mathrm{i} J_{b}^{a} \xi^{b}\right) .
$$

For the case of relativistic fields, where $\xi^{a}$ is a square-integrable solution of the Klein-Gordon equation defined on a background space-time, this decomposition corresponds to splitting the fields into positive- and negative-frequency parts.

Note that $\xi_{+}^{a}$ and $\xi_{-}^{a}$ are complex eigenstates of the $J_{b}^{a}$ operator: $J_{b}^{a} \xi_{+}^{b}=+\mathrm{i} \xi_{+}^{a}$ and $J_{b}^{a} \xi_{-}^{b}=-\mathrm{i} \xi_{-}^{a}$. The Hermitian ( $J$-invariance) condition (1) implies that two vectors of the same type (for example, a pair of $J$-positive vectors) are orthogonal with respect to the metric $g_{a b}$. Thus, we have $g_{a b} \xi_{+}^{a} \eta_{+}^{b}=0$ for any pair $\xi_{+}^{a}, \eta_{+}^{a}$ of $J-$ positive vectors, and $g_{a b} \xi_{-}^{a} \eta_{-}^{b}=0$ for any pair $\xi_{-}^{a}, \eta_{-}^{a}$ of $J$-negative vectors.

For a real vector $\xi^{a}$ it follows from (4) that $\xi_{-}^{a}=\bar{\xi}_{+}^{a}$. We can also split a complex vector into $J$-positive and $J$-negative parts. However, in splitting a complex vector $\zeta^{a}=\zeta_{+}^{a}+\zeta_{-}^{a}$ there is no a priori relationship between the components $\zeta_{+}^{a}$ and $\zeta_{-}^{a}$. Thus, if $\zeta^{a}$ is not real, then $\zeta_{-}^{a} \neq \overline{\zeta_{+}^{a}}$. The complex conjugate of a $J$-positive vector is nevertheless a $J$-negative vector, and vice versa. To be precise, we have $\overline{\zeta_{+}^{a}}=\bar{\zeta}_{-}^{a}$. 
Introducing $J$-positive and $J$-negative vectors allows us to express the Dirac inner product (3) in a simplified form, namely, $\langle\eta \mid \xi\rangle=\eta_{-}^{a} g_{a b} \xi_{+}^{b}$. The equivalence of (3) and the simplified form is verified by using (1) and the antisymmetry of $\Omega_{a b}$ :

$$
\begin{aligned}
\eta_{-}^{a} g_{a b} \xi_{+}^{b} & =\frac{1}{4}\left(\eta^{a}+\mathrm{i} J_{c}^{a} \eta^{c}\right) g_{a b}\left(\xi^{b}-\mathrm{i} J_{d}^{b} \xi^{d}\right) \\
& =\frac{1}{4}\left(g_{a b}+J_{a}^{c} J_{b}^{d} g_{c d}\right) \eta^{a} \xi^{b}-\frac{1}{4} \mathrm{i}\left(g_{a c} J_{b}^{c}-J_{a}^{c} g_{b c}\right) \eta^{a} \xi^{b} \\
& =\frac{1}{2} \eta^{a}\left(g_{a b}-\mathrm{i} \Omega_{a b}\right) \xi^{b} .
\end{aligned}
$$

\section{Quantum-mechanical observables}

We now explain how the observables of standard quantum mechanics are represented in terms of the geometry of the real Hilbert space $\mathrm{H}$. A quantum observable corresponds to a real symmetric $J$-invariant quadratic form on $\mathbb{H}$; that is, a real tensor $F_{a b}$ satisfying $F_{a b}=F_{b a}$ and $F_{a b} J_{c}^{a} J_{d}^{b}=F_{c d}$. The observable corresponding to the identity is $g_{a b}$, and for the expectation of $F$ in the state $\xi^{a}$ we write $\langle\xi|F| \xi\rangle /\langle\xi \mid \xi\rangle=F_{a b} \xi^{a} \xi^{b} / g_{a b} \xi^{a} \xi^{b}$. In general, for the states $\xi^{a}$ and $\eta^{a}$, we have $\langle\eta|F| \xi\rangle=\eta_{-}^{a} F_{a b} \xi_{+}^{b}$. The operator associated with the observable $F_{a b}$ is obtained by raising one of the indices with the inverse of the metric: $F_{b}^{a}=g^{a c} F_{c b}$. From the $J$-invariance of $F_{a b}$, when the operator $F_{b}^{a}$ acts on a $J$-positive vector, the result is another $J$-positive vector. Alternative ways to write $\langle\eta|F| \xi\rangle$ are $\eta_{-}^{a} g_{a c} F_{b}^{c} \xi_{+}^{b}=F_{c}^{a} \eta_{-}^{c} g_{a b} \xi_{+}^{b}$, which expresses the self-adjointness of $F_{b}^{a}$ with respect to the given inner product.

What are the symmetries of the Hilbert space $\mathbb{H}$ ? Rotations of $\mathbb{H}$ around the origin are given by orthogonal transformations, that is, the matrix operations $\xi^{a} \rightarrow$ $M_{b}^{a} \xi^{b}$ such that $g_{a b} M_{c}^{a} M_{d}^{b}=g_{c d}$. Such transformations preserve the norm $g_{a b} \xi^{a} \xi^{b}$ of the state $\xi^{a}$. The unitary group consists of orthogonal matrices that also leave the symplectic structure invariant: $\Omega_{a b} M_{c}^{a} M_{d}^{b}=\Omega_{c d}$.

In the case of an infinitesimal orthogonal transformation $M_{b}^{a}=\delta_{b}^{a}+\varepsilon f_{b}^{a}$, with $\varepsilon^{2} \sim 0$, it is easy to verify that $f_{b}^{a}$ must satisfy $g_{a c} f_{b}^{c}+g_{b c} f_{a}^{c}=0$, from which we deduce that $f_{b}^{a}$ has the form $f_{b}^{a}=g^{a c} f_{c b}$, where $f_{a b}$ is antisymmetric. For $M_{b}^{a}$ to be a unitary operator it is necessary and sufficient that $f_{a b}$ be $J$-invariant. We thus see that any infinitesimal unitary transformation has the form

$$
M_{b}^{a}=\delta_{b}^{a}+\varepsilon J_{c}^{a} F_{b}^{c},
$$

where $F_{b}^{a}$ is the operator associated with a standard quantum observable $F_{a b}$. Indeed, $f_{a b}$ is antisymmetric and $J$-invariant if and only if it can be expressed as

$$
f_{a b}=F_{a c} J_{b}^{c}
$$

where $F_{a b}$ is symmetric and $J$-invariant. If $F_{b}^{a}$ is proportional to the identity $g_{b}^{a}$ then (6) corresponds to an infinitesimal phase transformation. Conversely, if $F_{b}^{a}$ is trace-free, then (6) gives an infinitesimal special unitary transformation. 
Thus, we see how the operator $F_{b}^{a}$ is associated with both the observable $F_{a b}$ as well as the infinitesimal unitary transformation $\xi^{a} \rightarrow \xi^{a}+\varepsilon J_{b}^{a} F_{c}^{b} \xi^{c}$. The complete trajectory of the unitary transformation associated with the operator $F_{b}^{a}$ can be obtained by exponentiating the infinitesimal transformation and writing

$$
\xi^{a}(t)=\left.\exp \left(t J_{c}^{b} F_{d}^{c} \xi^{d} \partial_{b}\right) \xi^{a}\right|_{\xi^{a}=\xi^{a}(0)},
$$

where $\partial_{b}=\partial / \partial \xi^{b}$. The differential operator appearing in the exponent can be written as $J_{c}^{b} F_{d}^{c} \xi^{d} \partial_{b}=\frac{1}{2}\left(\Omega^{a b} \partial_{b} F\right) \partial_{a}$, where $F(\xi)=F_{a b} \xi^{a} \xi^{b}$. Thus, the quadratic form $F_{a b} \xi^{a} \xi^{b}$ appears as the generator of a Hamiltonian vector field $X^{a}(\xi)=$ $\partial \xi^{a} / \partial t$ on $\mathbb{H}$ given by $\partial \xi^{a} / \partial t=\frac{1}{2} \Omega^{a b} \partial_{b} F(\xi)$. The trajectory of the one-parameter family of unitary transformations associated with the observable $F_{a b}$ is generated by the Hamiltonian vector field $\frac{1}{2} \Omega^{a b} \partial_{b} F(\xi)$. If $H(\xi)=H_{a b} \xi^{a} \xi^{b}$ denotes the quadratic function on $\mathbb{H}$ associated with the Hamiltonian of a standard quantum system, then the Schrödinger equation is

$$
\frac{\partial \xi^{a}}{\partial t}=\frac{1}{2} \Omega^{a b} \partial_{b} H
$$

We conclude that standard quantum mechanics can be described in terms of the geometry of a real vector space $\mathbb{H}$ equipped with a complex structure $J_{b}^{a}$, a positive definite quadratic form $g_{a b}$, and a compatible symplectic structure $\Omega_{a b}$. Observables are $J$-invariant quadratic forms on $\mathbb{H}$, and dynamical trajectories are the symplectic vector field on $\mathbb{H}$ generated by such forms. All these structures are intrinsic to standard quantum theory.

\section{Space-time reflection symmetry}

In standard quantum theory, we fix the complex tensor $J_{b}^{a}$ on the space $\mathbb{H}$ of real state vectors; the remaining structures, namely, the positive-definite quadratic form $g_{a b}$ and the symplectic structure $\Omega_{a b}$, are then chosen so as to satisfy the compatibility conditions. The compatibility conditions are useful for relativistic fields, that is, when we insist that the creation and annihilation operators satisfy canonical commutation relations [23]. For the quantum theory of a $\mathscr{P} \mathscr{T}$-symmetric Hamiltonian we introduce another quadratic form called parity $\pi_{a b}$ that for certain purposes replaces the metric $g_{a b}$ of the standard theory. The parity operator, whose properties are defined below, can only be introduced if the complex dimension of the space of $J$-positive vectors is even. Hence, the dimensionality of the underlying space of real state vectors associated with $\mathscr{P} \mathscr{T}$-symmetric quantum theory is a multiple of four rather than two. This is the sense in which $\mathscr{P} \mathscr{T}$ symmetry extends (complex) quantum mechanics further into the complex domain [3].

Let us define the properties of the parity operator $\pi_{b}^{a}$. In a general sense, this operator represents space reflection and satisfies the conditions of a standard ob- 
servable in quantum theory discussed in Sec. 3. Therefore, $\pi_{a b}=g_{a c} \pi_{b}^{c}$ is required to be real and symmetric and to satisfy the $J$-invariance condition $\pi_{a b} J_{c}^{a} J_{d}^{b}=\pi_{c d}$. This condition is equivalent to the commutation relation $\pi_{c}^{a} J_{b}^{c}=J_{c}^{a} \pi_{b}^{c}$. In addition, the parity operator is required to be trace-free,

$$
\pi_{a}^{a}=0
$$

and to satisfy the orthogonality condition

$$
g_{a b} \pi_{c}^{a} \pi_{b}^{c}=g_{c d} .
$$

Thus, $\pi_{b}^{a}$ is unitary on the space of $J$-positive vectors associated with $\mathbb{H}$.

One may relax the trace-free condition (9) to define a generalization of the parity operator [24] on a complex Hilbert space of any dimension. For the physical intuition behind this, recall the case of a coupled pair of waveguides; here, $\mathscr{P}$ swaps the two waveguides. If there are three coupled waveguides, then a $\mathscr{P}$ reflection leaves the middle waveguide intact; there is a degenerate component. If such degeneracies are allowed, the parity operator can be defined in arbitrary dimension. Here, we focus on the nondegenerate case. Conditions (9) and (10) then prevent us from defining a parity structure unless the dimension of the underlying real Hilbert space $\mathbb{H}$ is a multiple of four. In particular, from the defining conditions of the parity operator half of its eigenvalues are +1 and the other half are -1 , and the parity operator is unique up to unitary transformations. The eigenvalues are \pm 1 because $\pi_{a b}$ is symmetric and the orthogonality condition (10) can be written as

$$
\pi_{c}^{a} \pi_{b}^{c}=\delta_{b}^{a}
$$

Since a successive application of space reflection is the identity, if we diagonalize $\pi_{b}^{a}$, the diagonal entries are \pm 1 , and the trace-free condition (9) implies that the two signs occur in equal numbers. Suppose that $\mathscr{P}$ and $\mathscr{P}^{\prime}$ are distinct parity operators. They have the same spectrum, so there exists a unitary transformation from one to the other. Hence, $\pi_{b}^{a}$ is unique up to unitary equivalence.

In $\mathscr{P} \mathscr{T}$-symmetric quantum theory we keep the real Hilbert space $\mathbb{H}$ and its complex structure $J_{b}^{a}$ and introduce a new inner-product on $\mathbb{H}$ in terms of the parity operator. The $\mathscr{P} \mathscr{T}$ inner product $\langle\eta \| \xi\rangle$ between the elements $\xi^{a}$ and $\eta^{a}$ in $\mathbb{H}$ is

$$
\langle\eta \| \xi\rangle=\frac{1}{2} \eta^{a}\left(\pi_{a b}-\mathrm{i} \omega_{a b}\right) \xi^{b},
$$

where $\omega_{a b}$ is defined by $\omega_{a b}=\Omega_{a c} \pi_{b}^{c}$. Equivalently, from (2) we can write $\omega_{a b}=$ $\pi_{a c} J_{b}^{c}$. Since $\pi_{a b}$ is an observable in standard quantum mechanics, $\omega_{a b}$ is antisymmetric and defines a new symplectic structure on $\mathbb{H}$ that is compatible with the complex structure $J_{b}^{a}$. One can easily verify the $J$-invariance condition $\omega_{a b} J_{c}^{a} J_{d}^{b}=\omega_{c d}$ associated with the symplectic structure.

As in standard quantum mechanics, the $\mathscr{P} \mathscr{T}$ inner product (12) can be written in terms of the $J$-positive and $J$-negative parts of the vectors $\xi^{a}$ and $\eta^{a}$. Splitting $\mathbb{H}$ into $J$-positive and $J$-negative parts depends on the complex structure $J_{b}^{a}$, and not on the 
associated quadratic forms. A short calculation then shows that $\langle\eta \| \xi\rangle=\eta_{-}^{a} \pi_{a b} \xi_{+}^{b}$. Indeed, starting from this equation we have $\eta_{-}^{a} \pi_{a b} \xi_{+}^{b}=\frac{1}{4}\left(\eta^{a}+\mathrm{i} J_{c}^{a} \eta^{c}\right) \pi_{a b}\left(\xi^{b}-\right.$ $\mathrm{i} J_{d}^{b} \xi^{d}$ ) by virtue of (5). Then, using of the $J$-invariance of $\pi_{a b}$ and the antisymmetry of $\omega_{a b}$, we are immediately led back to the inner product (12).

Because of (11), if we adapt the usual convention of writing $\pi^{a b}=g^{a c} g^{b d} \pi_{c d}$, then $\pi^{a b}$ is the inverse of $\pi_{a b}$ because $\pi_{a b} \pi^{b c}=\delta_{a}^{c}$. Analogously, the tensor $\omega^{a b}=$ $g^{a c} g^{b d} \omega_{c d}$ satisfies $\omega^{a b}=\pi^{a c} \pi^{b d} \omega_{c d}$ and $\omega^{a b}$ is the inverse of $\omega_{a b}$, so $\omega_{a b} \omega^{b c}=\delta_{a}^{c}$. Note also that $\omega_{a b}$ is $\mathscr{P}$-invariant because $\pi_{a}^{c} \pi_{b}{ }^{d} \omega_{c d}=\omega_{a b}$.

To summarize these results, in the case of the Hermitian theory we have the compatible structures $\left(J_{b}^{a}, g_{a b}, \Omega_{a b}\right)$ on $\mathbb{H}$, whereas the $\mathscr{P} \mathscr{T}$-symmetric quantum theory comes equipped with the compatible structures $\left(J_{b}^{a}, \pi_{a b}, \omega_{a b}\right)$. The key difference between the two theories is that, while $g_{a b}$ is positive definite, $\pi_{a b}$ is indefinite with the split signature $(+, \cdots,+,-, \cdots,-)$. The $\mathscr{P} \mathscr{T}$ norm (pseudo-norm) of a state $\xi^{a}$, which is defined by

$$
\langle\xi \| \xi\rangle=\frac{1}{2} \pi_{a b} \xi^{a} \xi^{b},
$$

can be either positive or negative, and in some cases may also vanish.

We interpret the $\mathscr{P} \mathscr{T}$ norm as follows. Given any real element $\xi^{a}$ in $\mathbb{H}$ we can split this into its positive and negative parity parts by writing $\xi^{a}=\xi_{\oplus}^{a}+\xi_{\ominus}^{a}$, where $\xi_{\oplus}^{a}=\frac{1}{2}\left(\xi^{a}+\pi_{b}^{a} \xi^{b}\right)$ and $\xi_{\ominus}^{a}=\frac{1}{2}\left(\xi^{a}-\pi_{b}^{a} \xi^{b}\right)$. These vectors are eigenstates of the parity operator $\pi_{a b}$, satisfying $\pi_{b}^{a} \xi_{\oplus}^{b}=\xi_{\oplus}^{a}$ and $\pi_{b}^{a} \xi_{\ominus}^{b}=-\xi_{\ominus}^{a}$. In terms of the projection operators $\Pi_{\oplus b}^{a}=\frac{1}{2}\left(\delta_{b}^{a}+\pi_{b}^{a}\right)$ and $\Pi_{\ominus b}^{a}=\frac{1}{2}\left(\delta_{b}^{a}-\pi_{b}^{a}\right)$ onto positive- and negative-parity eigenstates, we have

$$
\pi_{b}^{a}=\Pi_{\oplus b}^{a}-\Pi_{\ominus b}^{a},
$$

where $\Pi_{\oplus b}^{a} \xi^{b}=\xi_{\oplus}^{a} \quad$ and $\quad \Pi_{\ominus b}^{a} \xi^{b}=\xi_{\ominus}^{a}$. Furthermore, because $\pi_{b}^{a}$ and $J_{b}^{a}$ commute, the positive-parity component of the $J$-positive part of a real vector $\xi^{a}$ agrees with the $J$-positive part of the positive-parity part of $\xi^{a}$, and similarly for other such combinations. We can now establish the following result for the $\mathscr{P} \mathscr{T}$ norm.

Proposition 1 The squared $\mathscr{P} \mathscr{T}$ norm of a state $\xi^{a} \in \mathbb{H}$ is the difference between the squared Hermitian norm of the positive-parity part $\xi_{\oplus}^{a}$ of the state and that of the negative-parity part $\xi_{\ominus}^{a}$ of the state:

$$
\langle\xi \| \xi\rangle=\left\langle\xi_{\oplus} \mid \xi_{\oplus}\right\rangle-\left\langle\xi_{\ominus} \mid \xi_{\ominus}\right\rangle .
$$

Thus, if a state is "more probably" of positive parity, its $\mathscr{P} \mathscr{T}$-norm is positive. Conversely, for a state of "more probably" negative parity, its $\mathscr{P} \mathscr{T}$ norm is negative. The identity (15) follows immediately if we insert (14) into (13).

Finally, we note that if $\xi^{a}$ and $\eta^{a}$ are positive- and negative-parity states, then their standard quantum transition amplitude vanishes: $\left\langle\xi_{\oplus} \mid \eta_{\ominus}\right\rangle=0$. This follows 
from (3) if we insert $\eta_{\ominus}^{a}$ for $\eta^{a}$ and $\xi_{\oplus}^{a}$ for $\xi^{a}$ and use the identities $g_{a b} \Pi_{\oplus c}^{a} \Pi_{\ominus d}^{b}=0$ and $\Omega_{a b} \Pi_{\oplus c}^{a} \Pi_{\ominus d}^{b}=0$. The second of these two relations follows from the former because the $J$-tensor commutes with the parity projection operators.

\section{Observables and symmetries}

What are the transformations of $\mathbb{H}$ that preserve the $\mathscr{P} \mathscr{T}$ norm $\pi_{a b} \xi^{a} \xi^{b}$ ? The transformation $\xi^{a} \rightarrow M_{b}^{a} \xi^{b}$ preserves the $\mathscr{P} \mathscr{T}$ norm for all $\xi^{a} \in \mathbb{H}$ if and only if

$$
\pi_{a b} M_{c}^{a} M_{d}^{b} \xi^{c} \xi^{d}=\pi_{a b} \xi^{a} \xi^{b}
$$

for all $\xi^{a}$. In the case of the infinitesimal transformation $M_{b}^{a}=\delta_{b}^{a}+\varepsilon f_{c}^{a}$, (16) holds to first order in $\varepsilon$ if and only if

$$
\pi_{a b} f_{c}^{a} \xi^{b} \xi^{c}=0
$$

for all $\xi^{a}$, from which we deduce that $f_{b}^{a}$ must have the form

$$
f_{b}^{a}=\pi^{a c} f_{c b}
$$

where $f_{b c}$ is antisymmetric. As in Sec. $4, \pi^{a b}$ denotes the inverse of $\pi_{a b}$ and satisfies $\pi^{a b} \pi_{b c}=\delta_{c}^{a}$. Note that $\pi^{a b}$ is defined unambiguously without reference to $g_{a b}$.

To verify (18) we argue that if (17) holds for all $\xi^{a}$, then $\pi_{a b} f_{c}^{b}$ is antisymmetric. Writing $\pi_{a b} f_{c}^{b}=f_{a c}$, we obtain (18) by applying the inverse of $\pi_{a b}$ to each side. Thus, the infinitesimal pseudo-orthogonal transformations that preserve the $\mathscr{P} \mathscr{T}$ norm are given by $M_{b}^{a}=\delta_{b}^{a}+\varepsilon \pi^{a c} f_{c b}$, where $f_{a b}$ is antisymmetric.

We require that the transformation preserve the $\mathscr{P} \mathscr{T}$ symplectic structure $\omega_{a b}$. Because of the compatibility condition this is equivalent to requiring that complex structure is preserved. We have $\omega_{a b} M_{c}^{a} M_{d}^{b}=\omega_{c d}+\varepsilon\left(\omega_{a d} \pi^{a e} f_{e c}+\omega_{c b} \pi^{b e} f_{e d}\right)$ to first order in $\varepsilon$. Thus, in order for $\omega_{a b}$ to be preserved we require that $\omega_{a d} \pi^{a e} f_{e c}+$ $\omega_{c b} \pi^{b e} f_{e d}=0$. However, since $\omega_{a b}=\pi_{a c} J_{b}^{c}$, this condition implies that $f_{a b}$ is $J$ invariant. Because $f_{a b}$ is antisymmetric and $J$-invariant, it can be written in the form $f_{a b}=F_{a c} J_{b}^{c}$, where $F_{a b}$ is a $J$-invariant symmetric quadratic form on $\mathbb{H}$.

We conclude that the general infinitesimal pseudo-unitary transformation preserving $\pi_{a b}$ and $\omega_{a b}$ has the form $M_{b}^{a}=\delta_{b}^{a}+\varepsilon \omega^{a c} F_{c b}$, where $F_{a b}$ is a standard quantum observable; it is symmetric and $J$-invariant. It is interesting to recall equation (7) and to note that the same $J$-invariant quadratic forms on $\mathbb{H}$ appear both in standard quantum theory and in $\mathscr{P} \mathscr{T}$-symmetric quantum theory.

Arguments analogous to those in Sec. 3 show that the trajectory of the pseudounitary transformation associated with the operator $F_{b}^{a}=\pi^{a c} F_{c b}$ has the form

$$
\xi^{a}(t)=\left.\exp \left(t \omega^{b c} F_{c d} \xi^{d} \partial_{b}\right) \xi^{a}\right|_{\xi^{a}=\xi^{a}(0)},
$$


where $\partial_{b}=\partial / \partial \xi^{b}$. Thus, if $F(\xi)=F_{a b} \xi^{a} \xi^{b}$ is the quadratic function on $\mathbb{H}$ associated with an observable $F_{a b}$, then the dynamical equation for the corresponding one-parameter family of pseudo-unitary transformations on $\mathbb{H}$ preserves the $\mathscr{P} \mathscr{T}$ inner product, and it can be expressed in Hamiltonian form: $\partial \xi^{a} / \partial t=\frac{1}{2} \omega^{a b} \partial_{b} F$. This contrasts with (8) for standard quantum mechanics.

\section{$6 \mathscr{P} \mathscr{T}$-symmetric Hamiltonian operators}

We now introduce the notion of observables invariant under $\mathscr{P} \mathscr{T}$ symmetry and consider the properties of $\mathscr{P} \mathscr{T}$-symmetric Hamiltonian operators. Unlike Hermiticity in conventional quantum mechanics, we demand here that the Hamiltonian be invariant under space-time reflection. In ordinary quantum mechanics we require the Hamiltonian operator $H_{b}^{a}$ to be real, $H_{b}^{a}=\bar{H}_{b}^{a}$, and $J$-invariant, $J_{b}^{a} H_{c}^{b} J_{d}^{c}=H_{d}^{a}$. A Hamiltonian that satisfies these conditions is Hermitian. Here, we keep the $J$ invariance, but replace the reality condition with another condition that has the physical interpretation of invariance under space-time reflection.

We have introduced the real vector space $\mathbb{H}$ and the complex structure $J_{b}^{a}$ and we have showed that this structure can be augmented in two ways, either by introducing the positive-definite symmetric quadratic form $g_{a b}$ and associated symplectic structure $\Omega_{a b}$, or by introducing the split-signature indefinite form $\pi_{a b}$ and associated symplectic structure $\omega_{a b}$. Here, we consider either the structure $\left(J_{b}^{a}, g_{a b}, \Omega_{a b}\right)$ or the structure $\left(J_{b}^{a}, \pi_{a b}, \omega_{a b}\right)$ (or both). We call the former the $g$-structure on $\mathbb{H}$ and the latter the $\pi$-structure on $\mathbb{H}$. First, we consider those aspects of the $\mathscr{P} \mathscr{T}$ symmetry that arise when we only have the $\pi$-structure on $\mathbb{H}$. We make no direct use of the parity operator $\pi_{b}^{a}=g^{a c} \pi_{c b}$ for now (because this involves $g_{a b}$ ) and we consider only the consequence of introducing a $\pi$-structure on $\mathbb{H}$.

Suppose that $\mathbb{H}$ is endowed with a $\pi$-structure, and let $H_{b}^{a}$ be a complex operator on $\mathbb{H}_{\mathbb{C}}$. Thus, $H_{b}^{a}=X_{b}^{a}+\mathrm{i} Y_{b}^{a}$, where $X_{b}^{a}$ and $Y_{b}^{a}$ are real. We assume that $H_{b}^{a}$ is $J$-invariant. Then $H_{b}^{a}$ is invariant under space-time reflection (is $\mathscr{P} \mathscr{T}$-symmetric) with respect to the given $\pi$-structure, if

$$
\pi_{b c} \bar{H}_{d}^{c} \pi^{a d}=H_{b}^{a} .
$$

This relation suggests that if we take complex conjugate of the Hamiltonian followed by a parity transformation, then we recover the original Hamiltonian.

Next, we introduce the notion of a Hermitian form. A tensor $K_{a b}$ on $\mathbb{H}_{\mathbb{C}}$ is a Hermitian form if it is $J$-invariant and satisfies $\bar{K}_{a b}=K_{b a}$. Thus, $K_{a b}$ is a Hermitian form if $K_{a b}=X_{a b}+\mathrm{i} Y_{a b}$, where $X_{a b}$ and $Y_{a b}$ are real and $J$-invariant, and $X_{a b}$ is symmetric and $Y_{a b}$ is antisymmetric. Examples of Hermitian forms are $g_{a b}-\mathrm{i} \Omega_{a b}$ and $\pi_{a b}-\mathrm{i} \omega_{a b}$. The following proposition emerges from these definitions. 
Proposition 2 A Hamiltonian operator $H_{b}^{a}$ is $\mathscr{P} \mathscr{T}$-symmetric with respect to the $\pi$-structure $\left(J_{b}^{a}, \pi_{a b}, \omega_{a b}\right)$ if and only if there exists a Hermitian form $K_{a b}$ such that $H_{b}^{a}=\pi^{a c} K_{b c}$.

To verify Proposition 2 we note that $\pi_{b c} \bar{H}_{d}^{c} \pi^{a d}=\pi_{b c} \pi^{c e} \bar{K}_{d e} \pi^{a d}=\delta_{b}^{e} K_{e d} \pi^{a d}=$ $H_{b}^{a}$. Thus, $\mathscr{P} \mathscr{T}$ invariance of a Hamiltonian is an unconventional Hermiticity condition. One can characterize $\mathscr{P} \mathscr{T}$ invariance without reference to any elements of the $g$-structure on $\mathbb{H}$.

We now turn to the spectrum of the operator $H_{b}^{a}$, still keeping in the context of the $\pi$-structure. Because $H_{b}^{a}$ is complex, we must admit the possibility of complex eigenvectors, that is, elements of $\mathbb{H}_{\mathbb{C}}$. We define the $\mathscr{P} \mathscr{T}$ norm as follows: if $\phi^{a}$ is an element of $\mathbb{H}_{\mathbb{C}}$, then its $\mathscr{P} \mathscr{T}$ norm is $\pi_{a b} \phi^{a} \bar{\phi}^{b}$, which is the sum of the $\mathscr{P} \mathscr{T}$ norms of the real and imaginary parts of $\phi^{a}$.

Proposition 3 If the $\mathscr{P} \mathscr{T}$ norm of an eigenvector of a $\mathscr{P} \mathscr{T}$-symmetric Hamiltonian is nonvanishing, then the corresponding eigenvalue is real.

Proof. For some possibly complex value of $E$ the vector $\phi^{a}$, which may be complex, satisfies the eigenvalue equation $H_{b}^{a} \phi^{b}=E \phi^{a}$. Taking the complex conjugate, we have $\bar{H}_{b}^{a} \bar{\phi}^{b}=\bar{E} \bar{\phi}^{a}$. Transvecting each side of these equations with $\pi_{c a}$, we get $\pi_{c a} H_{b}^{a} \phi^{b}=E \pi_{c a} \phi^{a}$ and $\pi_{c a} \bar{H}_{b}^{a} \bar{\phi}^{b}=\bar{E} \pi_{c a} \bar{\phi}^{a}$. Hence, from Proposition 1 we get

$$
K_{a b} \phi^{b}=E \pi_{a b} \phi^{b}
$$

and $\bar{K}_{a b} \bar{\phi}^{b}=\bar{E} \pi_{a b} \bar{\phi}^{b}$, and because $K_{a b}$ is a Hermitian form we can replace this with

$$
K_{a b} \bar{\phi}^{b}=\bar{E} \pi_{a b} \bar{\phi}^{b} .
$$

Contracting (20) and (21) with $\bar{\phi}^{a}$ and $\phi^{a}$ and subtracting, we get $(E-\bar{E}) \pi_{a b} \phi^{a} \bar{\phi}^{b}=$ 0 , from which Proposition 3 follows.

Thus, if a $\mathscr{P} \mathscr{T}$-symmetric Hamiltonian has any complex eigenvalues, then the corresponding eigenstates have vanishing $\mathscr{P} \mathscr{T}$ norm. We proceed to augment the vector space $\mathbb{H}$ with the $g$-structure in addition to the $\pi$-structure. Introducing the $g$-structure allows us to consider the parity operator $\pi_{b}^{a}$. The condition (19) for the invariance under space-time reflection can now be written in the form

$$
\pi_{c}^{a} \bar{H}_{d}^{c} \pi_{b}^{d}=H_{b}^{a} .
$$

Note that real part of the Hamiltonian has even parity and imaginary part has odd parity. Therefore, writing $H_{b}^{a}=X_{b}^{a}+\mathrm{i} Y_{b}^{a}$, where $X_{b}^{a}$ and $Y_{b}^{a}$ are real, we get $\pi_{c}^{a} X_{d}^{c} \pi_{b}^{d}=X_{b}^{a}$ and $\pi_{c}^{a} Y_{d}^{c} \pi_{b}^{d}=-Y_{b}^{a}$. Conversely, any such complex operator is in- 
variant under space-time reflection. We then have the following observation on the reality of the energy eigenvalues.

Proposition 4 Let $E$ be an eigenvalue of a $\mathscr{P} \mathscr{T}$-symmetric Hamiltonian operator $H_{b}^{a}$ with corresponding eigenstate $\phi^{a}$. Then, $\bar{E}$ is also an eigenvalue of $H_{b}^{a}$, for which the associated eigenstate is $\pi_{b}^{a} \bar{\phi}^{b}$. In particular, if $\phi^{a}$ is a simultaneous eigenstate of the $\mathscr{P} \mathscr{T}$ operator, then $E$ is real.

Proof. Consider the eigenvalue equation

$$
H_{b}^{a} \phi^{b}=E \phi^{a},
$$

where $E$ is an energy eigenvalue, which may or may not be real. Substituting (22) in the right side of (23) gives $\pi_{c}^{a} \bar{H}_{d}^{c} \pi_{b}^{d} \phi^{b}=E \phi^{a}$. Taking the complex conjugate, we obtain $\pi_{c}^{a} H_{d}^{c} \pi_{b}^{d} \bar{\phi}^{b}=\bar{E} \bar{\phi}^{a}$. We then multiply on the left by the parity operator:

$$
H_{b}^{a} \pi_{c}^{b} \bar{\phi}^{c}=\bar{E} \pi_{b}^{a} \bar{\phi}^{b} .
$$

Thus, if $\phi^{a}$ is an energy eigenstate with eigenvalue $E$, then the state defined by $\pi_{b}^{a} \bar{\phi}^{b}$ is another energy eigenstate with eigenvalue $\bar{E}$. If, in addition, the energy eigenstate $\phi^{a}$ is a simultaneous eigenstate of the $\mathscr{P} \mathscr{T}$ operator, then $\pi_{b}^{a} \bar{\phi}^{b}=\lambda \phi^{a}$, where $\lambda$ is a pure phase. Substituting this into (24) and subtracting the result from (23) gives $\bar{E}=E$. This establishes Proposition 4 .

If an energy eigenstate $\phi_{i}^{a}$ is not a simultaneous eigenstate of $\mathscr{P} \mathscr{T}$, we say that $\mathscr{P} \mathscr{T}$ symmetry is broken. In this case the nonreal eigenvalues $E_{i}$ form complex conjugate pairs. If the $\mathscr{P} \mathscr{T}$ symmetry is unbroken so that $\left\{\phi_{i}^{a}\right\}$ are eigenstates of $\mathscr{P} \mathscr{T}$, the corresponding energy eigenvalues are real. Proposition 5 gives a sufficient (but not necessary) condition for the orthogonality of the eigenstates.

Proposition 5 If the eigenstates $\left\{\phi_{i}^{a}\right\}$ of a $\mathscr{P} \mathscr{T}$-symmetric Hamiltonian $H_{b}^{a}$ are also eigenstates of $\mathscr{P} \mathscr{T}$, then a sufficient condition for orthogonality of the eigenstates with respect to $\mathscr{P} \mathscr{T}$ inner-product is that the quadratic form $H_{a b}=g_{a c} H_{b}^{c}$ be symmetric.

Proof. Consider for $i \neq j$ a pair of eigenvalue equations $H_{b}^{a} \phi_{i}^{b}=E_{i} \phi_{i}^{a}$ and $H_{b}^{a} \phi_{j}^{b}=E_{j} \phi_{j}^{a}$. Transvect these equations with $\pi_{a c} \bar{\phi}_{j}^{c}$ and $\pi_{a c} \bar{\phi}_{i}^{c}$ and subtract:

$$
\bar{\phi}_{j}^{c} \pi_{c a} H_{b}^{a} \phi_{i}^{b}-\bar{\phi}_{i}^{c} \pi_{c a} H_{b}^{a} \phi_{j}^{b}=\pi_{a b}\left(E_{i} \phi_{i}^{b} \bar{\phi}_{j}^{a}-E_{j} \phi_{j}^{b} \bar{\phi}_{i}^{a}\right) .
$$

If the energy eigenstates are eigenstates of the $\mathscr{P} \mathscr{T}$ operator so that $\pi_{b}^{a} \bar{\phi}_{i}^{b}=$ $\phi_{i}^{a}$, then $\pi_{a b} \bar{\phi}_{i}^{b}=g_{a b} \phi_{i}^{b}$. Therefore, the left side of (25) becomes $\phi_{j}^{c} g_{c a} H_{b}^{a} \phi_{i}^{b}-$ 
$\phi_{i}^{c} g_{c a} H_{b}^{a} \phi_{j}^{b}=H_{c b}\left(\phi_{j}^{c} \phi_{i}^{b}-\phi_{i}^{c} \phi_{j}^{b}\right)$, where $H_{c b}=g_{c a} H_{b}^{a}$. Hence, the condition $H_{c b}=$ $H_{b c}$ ensures that the right side of (25) vanishes. This establishes Proposition 5.

Note that the symmetric condition on the complex Hamiltonian $H_{a b}$ is sufficient to ensure the orthogonality of the eigenstates, but it is not necessary.

\section{Charge-conjugation symmetry}

We have shown how to formulate quantum mechanics on a Hilbert space endowed with the structure of space-time reflection symmetry. Owing to the property of the parity structure $\pi_{a b}$, we noted that the resulting state space is equipped with an indefinite metric having a split signature; half of the states have positive and half have negative $\mathscr{P} \mathscr{T}$ norm.

In standard quantum mechanics the norm is associated with the probabilistic interpretation of the theory. Therefore, the physical interpretation of the inner product defined in (12) is somewhat ambiguous. To remedy this ambiguity, Ref. [3] pointed out the existence of a new symmetry associated with Hamiltonians that are $\mathscr{P} \mathscr{T}$ symmetric (see also [4]). By using this symmetry, which has an interpretation similar to that of charge conjugation, it is possible to introduce new inner product on the vector space $\mathbb{H}_{\mathbb{C}}$ spanned by the eigenstates of $\mathscr{P} \mathscr{T}$-symmetric Hamiltonians such that all the eigenstates have positive-definite norm. With the aid of this symmetry the probabilistic aspects of quantum theory are restored. We refer to this symmetry as charge conjugation in a broad sense, and introduce here briefly the properties of the symmetry associated with the 'charge' operator $C_{b}^{a}$. We make the following observation.

Proposition 6 Let $H_{b}^{a}$ be a $\mathscr{P} \mathscr{T}$-symmetric Hamiltonian operator. If the $\mathscr{P} \mathscr{T}$ symmetry is not broken, the energy eigenvalues are real. Let $\left\{\phi_{n}^{a}\right\}$ denote a set of normalized eigenstates of $H_{b}^{a}$. Then, the $\mathscr{P} \mathscr{T}$ inner product between a pair of energy eigenstates is $\left\langle\phi_{m} \| \phi_{n}\right\rangle=g_{a b} \phi_{n}^{a} \phi_{m}^{b}$.

The $\mathscr{P} \mathscr{T}$ inner product between a pair of states is given by $\pi_{a b} \phi_{n}^{a} \bar{\phi}_{m}^{b}$; then, the above equation follows from Proposition 3, which states that in unbroken $\mathscr{P} \mathscr{T}$ symmetry $\phi_{n}^{a}$ is an eigenstate of the $\mathscr{P} \mathscr{T}$ operator. Thus, we have $\pi_{a b} \phi_{n}^{a} \bar{\phi}_{m}^{b}=$ $g_{a c} \pi_{b}^{c} \phi_{n}^{a} \bar{\phi}_{m}^{b}=g_{a c} \phi_{n}^{a} \phi_{m}^{b}$. Because the $\mathscr{P} \mathscr{T}$ norm of the energy eigenstates are real, the real part of $\phi_{n}^{a}$ is orthogonal to its imaginary part with respect to $g_{a b}$.

Next, we normalize energy eigenstates according to $\phi_{n}^{a} \rightarrow \phi_{n}^{a} /\left(g_{a b} \phi_{n}^{a} \phi_{n}^{b}\right)^{1 / 2}$ and assume, in what follows, that $\phi_{n}^{a}$ is normalized. Then, according to the discussion of Sec. 4, exactly half of the normalized energy eigenstates have $\mathscr{P} \mathscr{T}$ norm +1 , and the remaining half have $\mathscr{P} \mathscr{T}$ norm -1 . We order the levels so that

$$
g_{a b} \phi_{m}^{a} \phi_{n}^{b}=(-1)^{n} \delta_{n m}
$$


With these conventions, we define the charge conjugation operator $C_{b}^{a}$. First, $C_{b}^{a}$ is $\mathscr{P} \mathscr{T}$-symmetric; thus, there exists a positive Hermitian form $L_{a b}$ satisfying $\bar{L}_{a b}=$ $L_{b a}$ such that $C_{b}^{a}=\pi^{a c} L_{b c}$. Second, $C_{b}^{a}$ commutes with the Hamiltonian $H_{b}^{a}$, so the eigenstates $\left\{\phi_{n}^{a}\right\}$ of $H_{b}^{a}$ are simultaneous eigenstates of $C_{b}^{a}$. Third, the eigenvalues of $C_{b}^{a}$ are given by $C_{b}^{a} \phi_{n}^{b}=(-1)^{n} \phi_{n}^{a}$, where $\phi_{n}^{a}$ satisfies (26). Thus, $C_{b}^{a}$ commutes with the Hamiltonian $H_{b}^{a}$ and its eigenvalues are precisely the $\mathscr{P} \mathscr{T}$ norm of the corresponding eigenstates. Hence, $C_{b}^{a}$ is involutary, $C_{b}^{a} C_{c}^{b}=\delta_{c}^{a}$, and trace-free, so $C_{a}^{a}=0$. In the infinite-dimensional context, $\mathscr{C}$ has a position-space representation $[3,4] \mathscr{C}=\sum_{n} \phi_{n}(x) \phi_{n}(y)$, which is similar to the position-space representation for the parity operator $\mathscr{P}=\sum_{n}(-1)^{n} \phi_{n}(x) \phi_{n}(-y)$. [Here, $\left\{\phi_{n}(x)\right\}$ are eigenfunctions of the $\mathscr{P} \mathscr{T}$-symmetric Hamiltonian.]

Having defined the operator $C_{b}^{a}$, we introduce on the vector space $\mathbb{H}_{\mathbb{C}}$ the following inner product. If $\xi^{a}, \eta^{a} \in \mathbb{H}_{\mathbb{C}}$, their inner product $\langle\xi \mid \eta\rangle$ is defined by

$$
\langle\xi \mid \eta\rangle=g_{a c} C_{b}^{c} \pi_{d}^{b} \eta^{a \bar{\xi}^{d}} .
$$

In particular, $\left\langle\phi_{n} \mid \phi_{m}\right\rangle=g_{a c} C_{b}^{c} \pi_{d}^{b} \phi_{m}^{a} \bar{\phi}_{n}^{d}=g_{a c} C_{b}^{c} \phi_{m}^{a} \phi_{n}^{b}=(-1)^{n} g_{a b} \phi_{m}^{a} \phi_{n}^{b}=\delta_{n m}$. Thus, (27) defines a positive-definite inner product between elements of $\mathbb{H}_{\mathbb{C}}$. Here, to simplify notation, we make no distinction between the Dirac inner product defined in (3) and the inner product (27) with respect to the $\mathscr{C} \mathscr{P} \mathscr{T}$ conjugation. This is because (27) is a natural extension of (3); when the prescribed Hamiltonian is Hermitian, (27) reduces to the conventional Dirac inner product (3). We emphasize that the charge operator $C_{b}^{a}$ introduced here is not the conventional charge-conjugation operator (cf. Streater \& Wightman [25]). In conventional Hermitian quantum theory, the commutation relation between the charge operator $\mathscr{C}$ and the parity operator $\mathscr{P}$ is $\mathscr{C} \mathscr{P}=(-1)^{N} \mathscr{P} \mathscr{C}$, where $N$ is the Fermion number. Hence, these operators commute for Bosons and anticommute for Fermions. The operators $\mathscr{C}$ and $\mathscr{P}$ in this paper are distinct square roots of the identity operator, and when Hamiltonian is Hermitian, $\mathscr{C}$ becomes identical to $\mathscr{P}$ so that the $\mathscr{C} \mathscr{P} \mathscr{T}$ invariance condition reduces to the Hermiticity requirement [3].

Acknowledgements We thank H. F. Jones and R. F. Streater for stimulating discussions. DCB was supported by The Royal Society and CMB was supported by the U.S. Department of Energy while the work presented here was carried out.

\section{References}

1. C. M. Bender and S. Boettcher, Phys. Rev. Lett. 80, 5243 (1998).

2. C. M. Bender, M. V. Berry, and A. Mandilara, J. Phys. A: Math. Gen. 35, L467 (2002).

3. C. M. Bender, D. C. Brody, and H. F. Jones, Phys. Rev. Lett. 89, 270401 (2002).

4. A. Mostafazadeh, J. Math. Phys. 43, 3944 (2002).

5. A. Ruschhaupt, F. Delgado, and J. G. Muga, J. Phys. A: Math. Theor. 38, L171 (2005).

6. K. G. Makris, R. El-Ganainy, D. N. Christodoulides, and Z. H. Musslimani, Phys. Rev. Lett. 100, 103904 (2008).

7. S. Klaiman, U. Günther, and N. Moiseyev, Phys. Rev. Lett. 101, 080402 (2002). 
8. A. Guo, G. J. Salamo, D. Duchesne, R. Morandotti, M. Volatier-Ravat, V. Aimez, G. A. Siviloglou, and D. N. Christodoulides, Phys. Rev. Lett. 103, 093902 (2009).

9. C. E. Rüter, K. G. Makris, R. El-Ganainy, D. N. Christodoulides, and D. Kip, Nat. Phys. 6, $192(2010)$.

10. L. Ge, Y. D. Chong, S. Rotter, H. E. Türeci, A. D. Stone, Phys. Rev. A 84, 023820 (2011).

11. Y. D. Chong, L. Ge, and A. D. Stone, Phys. Rev. Lett. 106, 093902 (2011).

12. S. Bittner, B. Dietz, U. Günther, H. L. Harney, M. Miski-Oglu, A. Richter, and F. Schäfer, Phys. Rev. Lett. 108, 024101 (2012).

13. J. Schindler, A. Li, M. C. Zheng, F. M. Ellis, and T. Kottos, Phys. Rev. A 84, 040101 (2011).

14. B. Peng, S. K. Özdemir, F. Lei, F. Monifi, M. Gianfreda, G. L. Long, S. Fan, F. Nori, C. M. Bender, and L. Yang, Nat. Phys. 10, 394 (2014).

15. M. Liertzer, L. Ge, A. Cerjan, A. D. Stone, H. Türeci, and S. Rotter, Phys. Rev. Lett. 108, 173901 (2012).

16. D. C. Brody and L. P. Hughston, Proc. R. Soc. London A 454, 2445 (1998).

17. D. C. Brody and L. P. Hughston, Proc. R. Soc. London A 455, 1683 (1999).

18. D. C. Brody and L. P. Hughston, J. Geom. Phys. 38, 19 (2001).

19. C. M. Bender and P. D. Mannheim, Phys. Lett. A 374, 1616 (2010).

20. R. Geroch, Ann. Phys. (N.Y.) 62, 582 (1971).

21. G. W. Gibbons and H. J. Pohle, Nucl. Phys. B 410, 117 (1993).

22. R. M. Wald, Quantum Field Theory in Curved Spacetime and Black Hole Thermodynamics (Chicago Univ. Press, Chicago, 1994).

23. A. Ashtekar and A. Magnon, Proc. R. Soc. London A 346, 375 (1975).

24. C. M. Bender, P. N. Meisinger, and Q. Wang, J. Phys. A: Math. Gen. 36, 1029 (2003).

25. R. F. Streater and A. S. Wightman, PCT, Spin and Statistics, and all That (Benjamin, New York, 1964). 